\title{
Idiopathic frozen shoulder
}

CPD

\section{Shane P Brun}

\section{Background}

Shoulder pain and stiffness affects at least one-quarter of the Australian population, with the primary care physician seeing $95 \%$ of these patients. Idiopathic frozen shoulder affects $>250,000$ Australians, making it a significant burden on both the individual and society. The primary care physician plays a major part in recognising the condition and formulating an evidencebased management plan in conjunction with the physiotherapist.

\section{Objective}

This article provides the reader with an understanding of the natural history, pathophysiology, phases and clinical features of idiopathic frozen shoulder. It also outlines patients at risk of developing idiopathic frozen shoulder and addresses an evidence-based conservative approach to the management of this condition.

\section{Discussion}

The primary care physician plays a pivotal part in the identification and management of idiopathic frozen shoulder, with the vast majority of patients responding to conservative management. A shared care approach with a skilled physiotherapist is essential.
THIS ARTICLE presents some of the more recent evidence for the identification and management of idiopathic frozen shoulder in the primary care setting. The majority of patients with shoulder pain are seen and treated in primary care settings, ${ }^{1}$ placing the primary care physician in a pivotal role for the diagnosis and management of this condition.

\section{Epidemiology}

Shoulder pain, aching or stiffness is a daily problem for almost a quarter of the Australian population. ${ }^{2}$ Of those who have shoulder pain, aching or stiffness, there is a significant proportion who have recognised risk factors that predispose them to idiopathic frozen shoulder, such as diabetes, thyroid dysfunction, hypercholesterolaemia and hypertension. ${ }^{3}$ Frozen shoulder is thought to have an incidence of $3-5 \%$ in the general population and up to $20 \%$ in individuals with diabetes. ${ }^{4}$ The peak age is 56 years, and the condition occurs slightly more often in women than men. ${ }^{5}$ It is a rare diagnosis before the age of 35 years and is unusual in patients aged over 70 years. ${ }^{5,6}$

\section{What is frozen shoulder?}

Idiopathic, or primary, frozen shoulder is defined as 'a condition characterized

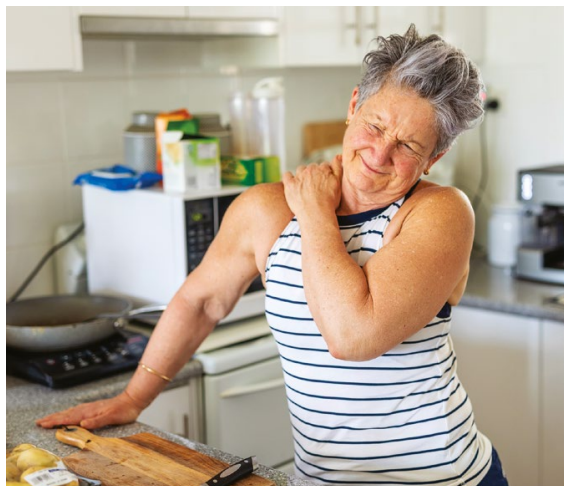

by functional restriction of both active and passive shoulder motion for which radiographs of the glenohumeral joint are essentially unremarkable except for the possible presence of osteopenia or calcific tendonitis'; ${ }^{7}$ the osteopenia results from limb and shoulder disuse. Primary frozen shoulder is a specific condition with an International Classification of Diseases, 10th Revision, code of M75.0; its aetiology is unclear, ${ }^{8}$ and it requires a management plan very different from other shoulder conditions such as osteoarthritis, rotator cuff pathology and subacromial bursitis. ${ }^{8}$

In line with many other shoulder conditions, a symptom of frozen shoulder is pain; however, it differs in that it also has mechanical restriction in all planes of movement of the glenohumeral joint. The limitation is due to glenohumeral capsular tightness and restriction without any evidence of adhesions. It occurs when the capsule, or the soft tissue envelope, becomes scarred and contracted, making the shoulder tight, painful and stiff. ${ }^{9}$

\section{What is the natural history?}

It is a general belief that frozen shoulder resolves spontaneously after $1-3$ years, ${ }^{9}$ although there is a paucity of long-term follow up data to support this. ${ }^{10} \mathrm{Up}$ to $40 \%$ of patients may have persistent 
symptoms, with $7-15 \%$ having some degree of permanent loss of movement. ${ }^{6,11}$ On the basis of a study of patients with frozen shoulder with a mean follow-up of 4.4 years, only $59 \%$ of patients had normal or near-normal shoulders as defined by no or minimal symptoms. ${ }^{6}$ Another study with a mean follow-up of seven years showed $50 \%$ of patients still had some mild pain and $60 \%$ had some ongoing stiffness. ${ }^{12}$ Subsequently, the potential chronicity of this problem is a burden to the patient and also a considerable cost to society in terms of health dollars, ${ }^{13}$ lost productivity and quality of life.

\section{Should investigations be performed for frozen shoulder?}

Frozen shoulder is predominantly a clinical diagnosis. ${ }^{8}$ Most imaging modalities and pathology testing that aim to confirm the diagnosis are usually unhelpful and may result in the wrong diagnosis being made. ${ }^{8,14}$ Investigations performed to exclude other pathologies may only occasionally be indicated, although the finding of a serious alternative diagnosis is rare. ${ }^{15}$

Once a diagnosis of frozen shoulder is made, it may be appropriate, with certain patient groups, to exclude the possibility of other conditions such as diabetes, thyroid dysfunction, hypercholesterolaemia and hypertension for which there is an increased incidence of idiopathic frozen shoulder. ${ }^{3}$

\section{The focused history}

Given that frozen shoulder is a clinical diagnosis, having a standardised and systematic focused history and focused examination strategy will be of significant advantage in differentiating frozen shoulder from other shoulder pathologies. ${ }^{16}$

Having a structured approach using appropriate questions is essential. ${ }^{16}$ Box 1 is an example of a suggested format to help rule in frozen shoulder and rule out alternative pathologies.

The patient presenting with idiopathic frozen shoulder is characterised by the spontaneous onset of pain with significant restriction of both active and passive range of movement of the shoulder. ${ }^{8}$ Confirming that external rotation is not possible with both active and passive movement is important in making the diagnosis. A key element in making the correct diagnosis is being able to differentiate limitation of shoulder movement secondary to pain, which is very common in most musculoskeletal conditions, versus limitation secondary to actual physical or mechanical restrictions. Differentiating between the two may be best achieved by performing passive movement of the glenohumeral joint. Limitation secondary to pain will be volunteered by the patient. Limitation due to capsular restriction will be felt by the examiner and patient, usually in the absence of pain. Missing this very subtle yet important difference is a clinical pitfall and is likely to result in overdiagnosis and misdiagnosis ${ }^{17}$ and subsequently an incorrect management plan.

Identifying how pain and disability from this condition affects the patient is important in helping to evaluate the patient's priorities, status, progress and outcome. Subsequently, as part of the focused history, the patient should be requested to perform the Shoulder Pain and Disability Index (SPADI; Appendix 1, available online; also available from most state and territory WorkSafe departments). ${ }^{18,19}$ The SPADI is a common and accepted assessment tool for shoulder function and pain and

\section{Box 1. Focused pain history for frozen shoulder}

Onset: The pain of idiopathic frozen shoulder is insidious, and there is no history of trauma. Location: Frozen shoulder pain is classically perceived around the region of deltoid insertion. Duration: The pain associated with frozen shoulder is persistent and may be chronic. Character: The pain associated with frozen shoulder is most often dull and aching. Aggravating and relieving factors: Pain is often worse at night and with extremes of movement; nonsteroidal anti-inflammatory drugs are often ineffective.

Radiation: Pain may radiate down the arm but rarely passes beyond the elbow. Timing: Pain is variable depending on the phase, although it may persist longer than four years. Severity: Pain is often severe in phase one and varies throughout the phases, progressively decreasing (although pain may not completely resolve). Using a visual analogue scale is useful to determine progress and as a baseline.

\begin{tabular}{|c|c|c|}
\hline Clinical phase & Description & Clinical details \\
\hline Phase one & $\begin{array}{l}\text { Painful phase or } \\
\text { pain-predominant } \\
\text { phase }\end{array}$ & $\begin{array}{l}\text { - Lasts } 2-9 \text { months } \\
\text { - Progressive stiffening and increasing pain on } \\
\text { movement and at night } \\
\text { - No history of shoulder trauma }\end{array}$ \\
\hline Phase two & $\begin{array}{l}\text { Stiffening, freezing } \\
\text { or stiffness } \\
\text { predominant phase }\end{array}$ & $\begin{array}{l}\text { - Lasts 4-12 months } \\
\text { - Gradual reduction of pain but stiffness persists } \\
\text { with considerable restriction in range of motion } \\
\text { - Pain gradually subsides but stiffness remains } \\
\text { - Pain may be apparent only at the extremes } \\
\text { of movement } \\
\text { - Gross reduction of glenohumeral movements, } \\
\text { with near total obliteration of external rotation }\end{array}$ \\
\hline Phase three & $\begin{array}{l}\text { Resolution or } \\
\text { thawing phase }\end{array}$ & $\begin{array}{l}\text { - Lasts } 12-42 \text { months } \\
\text { - Variable improvement in range of motion with } \\
\text { resolution of stiffness }\end{array}$ \\
\hline
\end{tabular}

*Time frames are approximations and all phases overlap by varying degrees. 
is used by many workers' compensation assessment authorities and rehabilitation services within Australia; therefore, familiarity by the primary care physician is a benefit.

Clinical presentation classically occurs in one of three overlapping phases (Table 1), ${ }^{8,14}$ and therefore the primary symptoms may vary depending on the phase of presentation.

\section{The focused examination}

As with the focused history, having a standardised and systematic approach to shoulder examination ensures accuracy in assessment and final diagnosis. ${ }^{16}$ It is important to be able to identify anatomical landmarks and appreciate both anatomical planes and ranges of movement for the shoulder joint. ${ }^{16}$ The focused shoulder examination involves the principles of inspection, palpation, movement and measuring (Table 2)..$^{5,7,8,16,20}$
The pathognomonic sign of frozen shoulder is almost complete loss of active and passive external rotation. ${ }^{8,20}$ As mentioned previously, it is essential to be able to differentiate between limited range of movement secondary to a mechanical block due to glenohumeral capsular tightness and restriction, and limitation secondary to pain.

\section{Management principles}

Only the conservative management principles of idiopathic frozen shoulder will be discussed in this article, as conservative management is the mainstay in the majority of cases, with surgery reserved for the most refractory. ${ }^{8}$

Each phase of frozen shoulder is managed differently and is dependent on the predominant signs and symptoms. There are four key management principles that overlap into all phases (Box 2), albeit the emphasis of each will vary depending on the phase.
Patient education regarding the natural history of the condition and proposed management plan are important and should always be included as part of the management plan. ${ }^{8,13}$ Understanding how the patient is affected by the condition is essential when formulating a management plan, and applying the SPADI is strongly recommended. ${ }^{18,19}$ The SPADI provides a baseline of current pain and disability, therefore allowing a serial measure of progress and how the condition affects the patient's quality of life and ability to work and function safely.

In all phases and indeed with all musculoskeletal conditions, optimising pain management is a priority. Without adequate and appropriate pain management, biomechanical changes occur secondary to guarding as a protective mechanism and altered limb use, which may result in muscle atrophy, osteopenia and tendinopathy.

Although patients may be reluctant to use appropriate analgesia, it is essential

\section{Table 2. Frozen shoulder examination findings}

\begin{tabular}{ll} 
Investigation & Examination findings \\
\hline Inspection & Mild disuse atrophy of the deltoid and supraspinatus may \\
be present. & It is a normal finding for the dominant shoulder to sit lower than \\
the non-dominant shoulder, subsequently this in itself is not \\
necessarily a pathological finding.
\end{tabular}

Palpation - There may be diffuse tenderness over the glenohumeral joint, which may extend to the trapezius and interscapular region; this is secondary to splinting of the painful shoulder.

- For the musculoskeletal system, pain is poorly localised yet tenderness is well localised, hence the patient has a perceived location of pain at or around the deltoid insertion, yet tenderness is identified over the glenohumeral joint.

Movement $\begin{aligned} & \text { This stage should be performed simultaneously using the } \\ & \text { and } \\ & \text { measuring }\end{aligned}$
rangected shoulder as the control for normal power and normal
r It is at this stage of the examination that limitation of range of
movement secondary to mechanical block rather than pain is
differentiated.
- With suspected frozen shoulder, both active and passive
movements must be performed.
- The pathognomonic sign of frozen shoulder is almost complete
loss of active and passive external rotation.
- Limitation to active and passive abduction, forward flexion and/
or internal rotation may also be found.

\section{Box 2. Conservative management principles}

1. Education
a. The nature of the condition
b. The natural history
c. The potential chronicity of the condition

2. Regular and breakthrough analgesia

a. Start with simple regular analgesia; care should be taken with regular and prolonged use of nonsteroidal anti-inflammatory drugs if these are used

b. Supplement with stronger breakthrough analgesia

c. Topical creams, lotions and ointments are ineffective, but their use may be reasonable if the patient perceives there is benefit and no harm is being done

3. Physiotherapy

a. Involve early

b. Intervention strategies will vary depending on the phase

4. Intra-articular corticosteroid injection

a. More effective the earlier it is given

b. Aseptic technique is necessary

c. Accuracy can be ensured using anatomical landmarks or image guidance

5. Other injection therapies

a. Hydrodilatation has been shown to be effective and safe

b. The addition of a small amount of local anaesthetic is beneficial 
that analgesia is prescribed regularly, with the availability of alternative additional analgesia for breakthrough pain. ${ }^{8}$

The involvement of a physiotherapist who has experience in the management of frozen shoulder is essential in each phase of the condition.

Intra-articular corticosteroid injections are safe and effective and have been shown to hasten improvement in pain and function. Many studies show benefit either alone or in conjunction with exercise program..$^{8,10,21,22}$

Glenohumeral joint injection performed in the early stages of frozen shoulder by the primary care physician, with the necessary skills and equipment, can provide significant improvement in physiotherapy and/or a home-based

passive range of movement. ${ }^{8,23}$ Figure 1 identifies the anatomical landmarks required to ensure the injection is located within the glenohumeral joint. If any doubt exists, the image-guided approach should be performed.

Another injection-therapy modality is hydrodilatation, which involves injecting the glenohumeral joint capsule with a mixture of sodium chloride $(0.9 \%)$ and corticosteroid made up to a volume of $40 \mathrm{~mL}$. This procedure results in stretching of the capsule in addition to providing the benefits of the injected steroid. The injection technique is the same as described previously.

Hydrodilatation has been shown to be effective when used alone or in combination with physiotherapy. ${ }^{10,17}$ The author has found the addition of
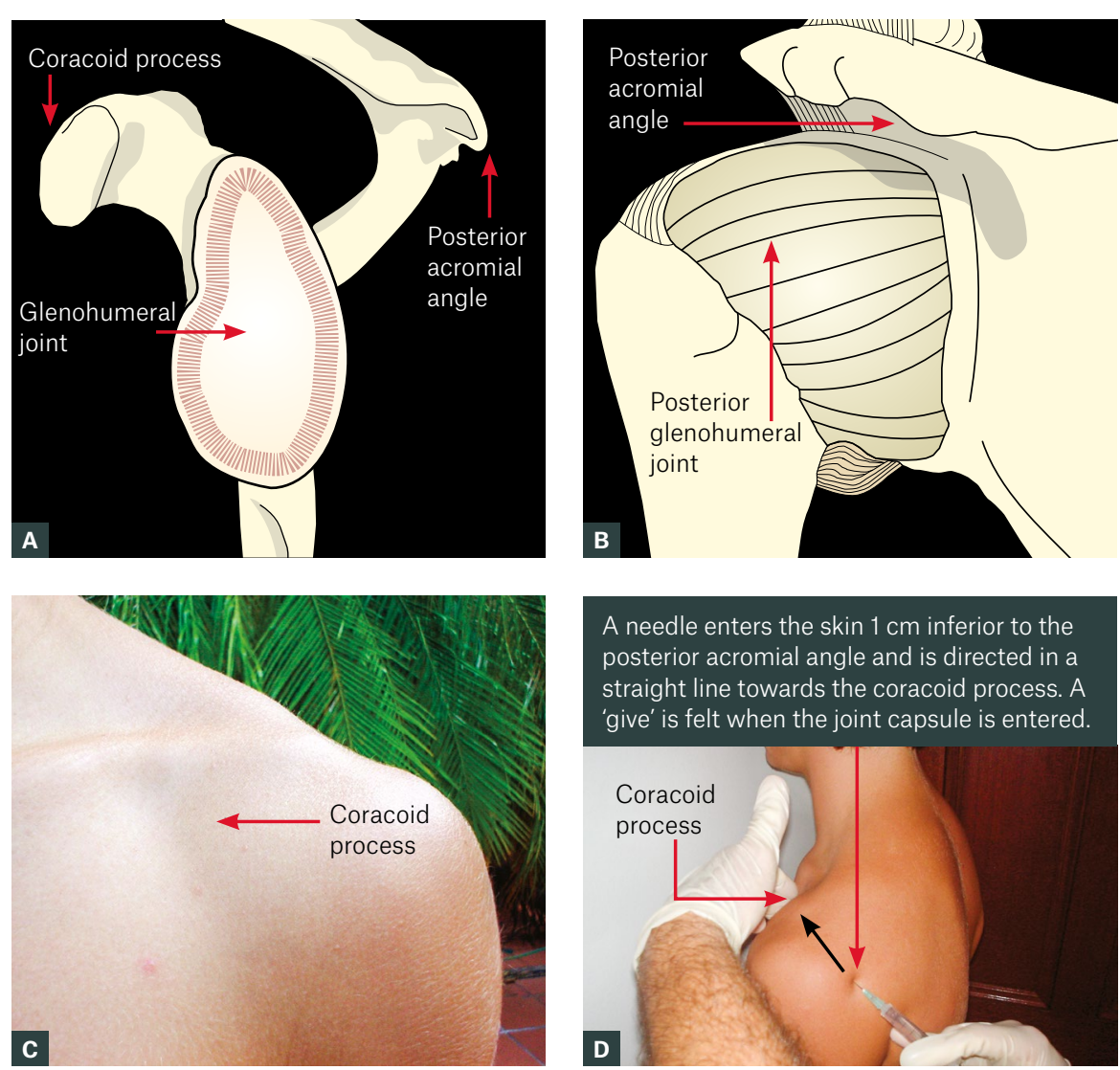

Figure 1. Anatomical landmarks for glenohumeral joint injection

A. Lateral view of glenohumeral joint with glenoid exposed; B. Posterior view of glenohumeral joint with capsule intact; C. Anatomical surface location of coracoid process; D. Surface anatomy depicting needle insertion point and direction with the glenohumeral joint being midway between posterior acromial angle and coracoid process
5-10 mL of $0.5 \%$ bupivacaine, included within the maximum volume of $40 \mathrm{~mL}$, to be effective in providing short-term pain relief and allowing for pain-free physiotherapy and exercises following this procedure. Prior to the procedure taking place, the usual medication administration safeguards must be adhered to including checking for allergies, medication contraindications, medication side effects and dosage recommendations.

The use of intra-articular sodium hyaluronate has been assessed in several randomised controlled trials and has been shown not to be effective in frozen shoulder. $^{22}$

The use of oral steroids has also been tested; to date, the benefits do not appear to outweigh the potential risk. ${ }^{22,24,25}$

\section{Conclusion}

Idiopathic frozen shoulder is a common presentation. It has three overlapping phases, each comprising pain, stiffness and limited range of movement in varying degrees. Pain and/or stiffness may become a chronic problem. To be able to differentiate between limited range of movement secondary to mechanical restriction and limited range of movement secondary to pain is an important principle. Most patients with idiopathic frozen shoulder are best managed conservatively. Involving a physiotherapist in the management plan is best practice. Appropriate oral analgesia is a mainstay of management. Intra-articular injections of the glenohumeral joint should always be considered in the early phases, especially if analgesia and physiotherapy are providing little-to-no benefit.

\section{Authors}

Shane P Brun MBBS, FFSEM (UK), FASMF, FACRRM, FRACGP, FARGP, MTrauma (Distinction), MSpMed, MEd, BAppSc, DCH, Associate Professor, College of Medicine and Dentistry, James Cook University, QLD; Visiting Professor, Sports Medicine Unit, University of Malaysia, MY; Senior Medical Officer and Instructor, Fédération Internationale de Football Association (FIFA), CH; Senior Medical Officer and Instructor, Asian Football Confederation (AFC), MY. shane.brun@jcu.edu.au

Competing interests: None.

Funding: None.

Provenance and peer review: Commissioned, externally peer reviewed. 


\section{References}

1. Artus M, van der Windt DA, Afolabi EK, et al. Management of shoulder pain by UK general practitioners (GPs): A national survey. BMJ Open 2017;7(6):e015711. doi: 10.1136/bmjopen-2016015711.

2. Hill CL, Gill TK, Shanahan EM, Taylor AW. Prevalence and correlates of shoulder pain and stiffness in a population-based study: The North West Adelaide Health Study. Int J Rheum Dis 2010;13(3):215-22. doi: 10.1111/j.1756185X.2010.01475.x

3. Milgrom $C$, Novack $V$, Weil $Y$, Jaber $S$, Radeva-Petrova DR, Finestone A. Risk factors for idiopathic frozen shoulder. Isr Med Assoc $J$ 2008;10(5):361-64.

4. Manske RC, Prohaska D. Diagnosis and management of adhesive capsulitis. Curr Rev Musculoskelet Med 2008;1(3-4):180-89. doi: 10.1007/s12178-008-9031-6.

5. Rizk TE, Pinals RS. Frozen shoulder. Semin Arthritis Rheum 1982;11(4):440-52

6. Hand C, Clipsham K, Rees JL, Carr AJ. Longterm outcome of frozen shoulder. J Shoulder Elbow Surg 2008;17(2):231-36. doi: 10.1016/j. jse.2007.05.009.

7. Zuckerman JD, Rokito A. Frozen shoulder: A consensus definition. J Shoulder Elbow Surg 2011;20(2):322-25. doi: 10.1016/j.jse.2010.07.008.

8. Dias R, Cutts $S$, Massoud S. Frozen shoulder. BMJ 2005;331(7530):1453-56. doi: 10.1136/ bmj.331.7530.1453.

9. Brealey S, Armstrong AL, Brooksbank A, et al. United Kingdom Frozen Shoulder Trial (UK FROST), multi-centre, randomised, 12 month, parallel group, superiority study to compare the clinical and cost-effectiveness of Early Structured Physiotherapy versus manipulation under anaesthesia versus arthroscopic capsular release for patients referred to secondary care with a primary frozen shoulder: Study protocol for a randomised controlled trial. Trials 2017;18(1):614. doi: 10.1186/s13063-017-2352-2.

10. Rangan A, Hanchard N, McDaid C. What is the most effective treatment for frozen shoulder? BMJ 2016;354:i4162. doi: 10.1136/bmj.i4162.

11. Hazleman BL. The painful stiff shoulder. Rheumatol Phys Med 1972;11(8):413-21. doi: 10.1093/rheumatology/11.8.413

12. Shaffer B, Tibone JE, Kerlan RK. Frozen shoulder. A long-term follow-up. J Bone Joint Surg Am 1992;74(5):738-46. doi: 10.1136/ard.43.3.361.
13. Jones S, Hanchard N, Hamilton S, Rangan A. A qualitative study of patients' perceptions and priorities when living with primary frozen shoulder. BMJ Open 2013;3(9):e003452. doi: 10.1136/ bmjopen-2013-003452.

14. Guyver PM, Bruce DJ, Rees JL. Frozen shoulder A stiff problem that requires a flexible approach. Maturitas 2014;78(1):11-16. doi: 10.1016/j. maturitas.2014.02.009.

15. Sano H, Hatori M, Mineta M, Hosaka M, Itoi E. Tumors masked as frozen shoulders: A retrospective analysis. J Shoulder Elbow Surg 2010;19(2):262-66. doi: 10.1016/j.jse.2009.05.010.

16. Brun S. Initial assessment of the injured shoulder. Aust Fam Physician 2012;41(4):217-20.

17. Robinson $\mathrm{CM}$, Seah $\mathrm{KT}$, Chee $\mathrm{YH}$, Hindle P, Murray IR. Frozen shoulder. J Bone Joint Surg Br 2012;94(1):1-9. doi: 10.1302/0301620X.94B1.27093.

18. Breckenridge JD, McAuley JH. Shoulder Pain and Disability Index (SPADI). J Physiother 2011;57(3):197. doi: 10.1016/S18369553(11)70045-5.

19. Thoomes-de Graaf M, Scholten-Peeters W, Duijn $\mathrm{E}$, et al. The responsiveness and interpretability of the Shoulder Pain and Disability Index. J Orthop Sports Phys Ther 2017;47(4):278-86. doi: 10.2519/jospt.2017.7079.

20. Wolf EM, Cox WK. The external rotation test in the diagnosis of adhesive capsulitis. Orthopedics 2010;33(5). doi: 10.3928/01477447-20100329-11.

21. Ryans I, Montgomery A, Galway R, Kernohan WG, McKane R. A randomized controlled trial of intra-articular triamcinolone and/or physiotherapy in shoulder capsulitis. Rheumatology (Oxford) 2005;44(4):529-35. doi: 10.1093/rheumatology/ keh535.

22. Maund E, Craig D, Suekarran S, et al. Management of frozen shoulder: A systematic review and cost-effectiveness analysis. Health Technol Assess 2012;16(11):1-264. doi: 10.3310/ hta16110.

23. Sun Y, Liu S, Chen S, Chen J. The effect of corticosteroid injection into rotator interval for early frozen shoulder: A randomized controlled trial. Am J Sports Med 2018;46(3):663-70. doi: $10.1177 / 0363546517744171$.

24. Buchbinder R, Green S, Youd JM, Johnston RV. Oral steroids for adhesive capsulitis. Cochrane Database Syst Rev 2006(4):CD006189. doi: 10.1002/14651858.CD006189.
25. Buchbinder R, Hoving JL, Green S, Hall S, Forbes A, Nash P. Short course prednisolone for adhesive capsulitis (frozen shoulder or stiff painful shoulder): A randomised, double blind, placebo controlled trial. Ann Rheum Dis 2004;63(11):1460-469. doi: 10.1136/ard.2003.018218. 